\title{
Network Snakes for the Segmentation of Adjacent Cells in Confocal Images
}

\author{
Matthias Butenuth ${ }^{1}$ and Fritz Jetzek ${ }^{2}$ \\ ${ }^{1}$ Institut für Photogrammetrie und GeoInformation, \\ Leibniz Universität Hannover, 30167 Hannover \\ ${ }^{2}$ Evotec Technologies GmbH, 22525 Hamburg \\ Email: butenuth@ipi.uni-hannover.de
}

\begin{abstract}
Network snakes constitute one of the latest advances in the research of image segmentation techniques: they integrate topology into active contour models. This concept is applied to the problem of delineating biological cells in confocal images and is compared to results obtained with a current state-of-the-art segmentation method. Lacking a representative gold standard for this task, we adjust the well-established measure of segmentation quality developed by Pratt to our requirements and discuss the matching result of network snakes on one hand and conventional algorithms on the other. The work concludes with a discussion of some characteristic features and flaws of the approach.
\end{abstract}

\section{Introduction}

The advent of high content screening facilities by means of image acquisition from confocal laser scanning microscopes has significantly changed the conditions of pharmaceutical target research in recent years. One of the needs that arose is the task of automatically segmenting cell culture images. Information relevant to biological research includes cell properties like shape, size, and intensity distribution, which use the exact boundaries of cells.

The general problem of image segmentation still remains largely unsolved with respect to the sophisticated demands of medical applications. With the availability of multi-channel fluorescence labelings, image segmentation can often be split into smaller problems, for example, by detecting cell nuclei in one channel first, and subsequently using the prior information for the segmentation of cells in the second channel. However, machine-dependent artifacts like noise and objecttypical characteristics like homogeneous areas in the intensity distribution at cell boundaries still provide major difficulties.

Enhancing well-known active contour models, which are only defined for closed object boundaries, a new methodology called network snakes [1] was introduced having the possibility to delineate objects, which form a network and thus interact during the optimization process. Cells are often adjacent with only one visible boundary in between. Therefore, network snakes can be used to detect the cell boundaries without intersection or overlap of the individual cells. 
The next section contains some thoughts about the segmentation and modeling of cells. In addition, network snakes are shortly summarized to provide a base for introducing topology to the traditional concept. In section 3 the methodology to delineate cells using network snakes is described. In order to evaluate the proposed method, a conventional method is used to derive a reference. Subsequently, both methods are validated with the measure of segmentation quality introduced by Pratt [2]. Finally, some concluding remarks are given.

\section{State of the art and new contribution}

Image analysis for cell segmentation still follows paradigms that have been developed years ago, the most prominent ones being global and local thresholding techniques and the watershed transform [3]. A lot of research has been performed on the topic, but the aspect of modeling the natural properties of the objects of interest is largely neglected, even though it is obvious that this would improve the segmentation process [4]. In contrary, the presented network snakes approach follows a modeling scheme in that it does not restrict the detection of boundaries to a mere analysis of local intensity distributions, but takes energy minimization considerations into account that correspond more closely to cytoplasmic membrane morphology of adjacent cells.

The total energy of a traditional snake, to be minimized, is defined as [5]

$$
E_{\text {snake }}^{*}=\int_{0}^{1} E_{\text {snake }}(v(s)) d s=\int_{0}^{1}\left(E_{\text {img }}(v(s))+E_{\text {int }}(v(s))\right) d s
$$

where $E_{\mathrm{img}}(v(s))$ represents the image energy and $E_{\text {int }}(v(s))$ the internal energy. A minimum of the total energy $E_{\text {snake }}^{*}$ can be derived by solving the respective Euler equations [5]. The derivatives are approximated with finite differences since they can not be computed analytically. The Euler equations read

$$
\begin{aligned}
& \alpha_{i}\left(v_{i}-v_{i-1}\right)-\alpha_{i+1}\left(v_{i+1}-v_{i}\right) \\
+ & \beta_{i-1}\left(v_{i-2}-2 v_{i-1}+v_{i}\right)-2 \beta_{i}\left(v_{i-1}-2 v_{i}+v_{i+1}+\beta_{i+1}\left(v_{i}-2 v_{i+1}+v_{i+2}\right)\right. \\
+ & f_{v}(v)=0
\end{aligned}
$$

and can be rewritten in matrix form as

$$
A v+f_{v}(v)=0
$$

Equation 3 can be solved iteratively by introducing a step size $\gamma$. Finally, a solution can be derived by matrix inversion

$$
v_{t}=(A+\gamma \mathrm{I})^{-1}\left(\gamma v_{t-1}-\kappa f_{v}\left(v_{t-1}\right)\right)
$$

where $\mathrm{I}$ is the identity matrix and $\kappa$ is an additional parameter in order to control the weight between internal and image energy. 
The minimization of the internal energy during the optimization process is only defined for closed object boundaries, i.e. $v_{0}=v_{n}[5]$. A new methodology was presented to overcome this limitation, called network snakes [1]. Integrating the topology into the energy minimization process causes a problem when solving equations 2 and 3: the derivatives approximated by finite differences are not defined for nodes with a degree $\rho(v) \neq 2$, because the required neighboring nodes are either not available or exist multiple times. A new definition of the total energy is proposed, which enables control at the common nodal point $v_{n}$, in that case with a degree $\rho(v)=3$ with $v_{n}=v_{a_{n}}=v_{b_{n}}=v_{c_{n}}[1]$

$$
\begin{aligned}
& \beta\left(v_{a_{n}}-v_{a_{n-1}}\right)-\beta\left(v_{a_{n-1}}-v_{a_{n-2}}\right)+f_{v_{a}}\left(v_{a}\right)=0 \\
& \beta\left(v_{b_{n}}-v_{b_{n-1}}\right)-\beta\left(v_{b_{n-1}}-v_{b_{n-2}}\right)+f_{v_{b}}\left(v_{b}\right)=0 \\
& \beta\left(v_{c_{n}}-v_{c_{n-1}}\right)-\beta\left(v_{c_{n-1}}-v_{c_{n-2}}\right)+f_{v_{c}}\left(v_{c}\right)=0
\end{aligned}
$$

$v_{a}, v_{b}$ and $v_{c}$ represent three contours, each ending in a common nodal point $v_{n}$. The energy definition of equation 5 allows for a minimization process to control the shape of each contour segment separately even though they end in one common point. The new definition is straightforward for nodes with a degree $\rho(v)=1$ and $\rho(v)>3$, which are not used in this work. Of course, the proposed method requires a given topology, which is assumed to be correct.

\section{Methods}

Two different segmentation methods are compared for delineating biological cells in confocal images. A method using network snakes is proposed on one hand and a conventional method is presented on the other. The strategy of both methods is divided into two parts: at first, the image representing the cell nuclei is used to derive a coarse initialization. In a second step, both techniques are applied to the cytoplasm image to obtain the cell boundaries.

Concerning the use of network snakes, the required initialization is accomplished by means of a segmentation of the relative homogeneous background in the cell nuclei image utilizing a region growing algorithm. Subsequently, a skeleton is computed to yield a coarse initial network representing the boundaries between adjacent cells. Since each cell nucleus is located within the associated cell membrane, the skeleton can be used to derive the topology of the network. At that time a somewhat inaccurate geometrical position is tolerated. In the second step of the strategy the initial boundaries between the cells are used to initialize the network snakes approach and to optimize the preliminary boundaries deriving the final results.

For reasons of comparison, we also apply a conventional segmentation algorithm $^{1}$ to the same data as follows [6]: The nuclei image is transformed into a binary image using a sliding window to compute local thresholds for the intensity distribution. The resulting binary image is assumed to contain all nuclei

\footnotetext{
${ }^{1}$ Acapella data analysis software, Evotec Technologies GmbH
} 
Fig. 1. Validation of the segmentation: (a) cell boundaries as calculated with network snakes, (b) corresponding result with conventional segmentation method, (c) Pratt values for all pairs of cell boundaries from both results

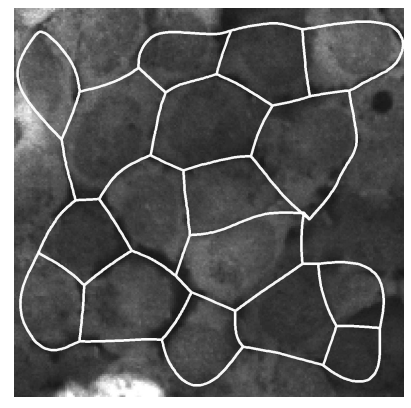

(a)

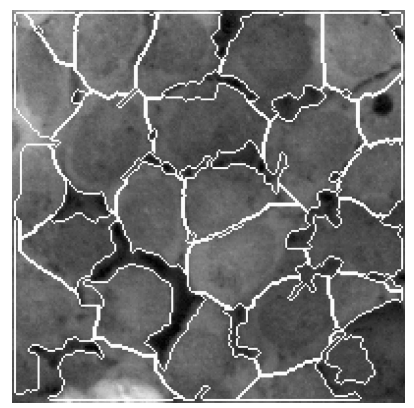

(b)

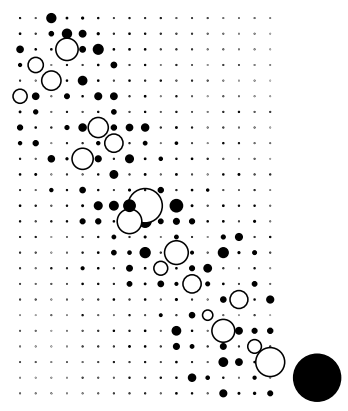

(c)

to be detected. A connected-component-labeling of this data yields a stencil of separate objects. Objects with low contrast are eliminated in order to remove artifacts erroneously detected as nuclei. The final step is carried out on the cytoplasm image exploiting the nuclei locations as seed objects for a watershed transform that delivers the final borders of the cytoplasm.

For the validation of the results a measure introduced by Pratt [2, pp. 497] is utilized, originally intended to evaluate the automatic detection of lines. The measure

$$
R=\frac{1}{I_{N}} \sum_{i=1}^{I_{A}} \frac{1}{1+\delta\left(x_{i}, I\right)}
$$

compares the distances $\delta\left(x_{i}, I\right)$ of claimed boundary pixels $x_{i} \in A$ to assumed ideal boundary pixels $I$ with $I_{N}=\max \left(I_{I}, I_{A}\right)$. Equation 6 yields a range of ]0..1] where values close to 0 correspond to high discrepancies and a value of 1 corresponds to an ideal matching of a pair of boundaries.

\section{Results}

Results of the proposed method using network snakes and using the conventional method are presented in figure 1. 17 cells are detected applying the network snakes approach (Fig. 1a), and 25 cells are segmented with the conventional watershed-based algorithm (Fig. 1b). Figure 1c illustrates the Pratt values for all pairs of cell boundaries from the two segmentation techniques. The 25 rows and 17 columns of the table correspond to the objects from the conventional and from the network snake approach, respectively. The Pratt values are given in circles, where larger circles correspond to a better match of the pair. To enhance the result, circle radii have been computed as $(1+R)^{2}-1$. In order to provide a scale, the black circle at the bottom right is given, which represents an ideal match $R=1$. Those circles that actually denote pairs of objects that belong to an identical cell are depicted with a white filling. 
Three important observations can be made from the analysis. First, those circles that denote correct correspondences are by far the largest; this means segmentation with network snakes performs well enough to identify cells. Of course, the underlying assumption is based on correct reference data contributed by the conventional method. Second, the matching scores are smaller than the ideal value of 1 . This distortion is due to the nature of both techniques: While conventional treatment of local intensities is bound to have a frayed border, the modeled shape behavior of snakes let boundaries appear smoother. However, it is this difference in the borders that decreases the interpretability of the Pratt value. Third, the proposed method using network snakes does not consider incomplete cells at the image boundary and, thus, less cells are segmented. Independently of image border effects any cells have been extracted successfully.

\section{Discussion}

This contribution compares a new segmentation technique of delineating adjacent cells in confocal images - the analysis with network snakes - to a typical conventional algorithm. It is known that the construction of a gold standard for segmentation evaluation is problematic. The used Pratt method is meaningful, even though it can not cover an overall set of assessment criteria. The comparison cannot conclude whether the candidate algorithm performs better than the standard but it can give an impression of its quality in terms of the state-of-the-art. Considering the vast differences that human testers produce in manually segmented images, we claim that the proposed method is justifiable. An important conclusion relates to the shape behavior of the cell boundaries: within the network snakes approach the modeled shape is represented in a smoother and more natural way compared to the watershed-based conventional approach, which could not be reflected in the evaluation in a positive manner.

\section{References}

1. Butenuth M. Segmentation of Imagery Using Network Snakes. International Archives of Photogrammetry, Remote Sensing and Spatial Information Sciences 2006;36(3):1-6.

2. Pratt WK. Digital Image Processing. Wiley-Interscience, New York; 1978.

3. Roerdink JBTM, Meijster A. The watershed transform: Definitions, algorithms and parallelization strategies. Fundamenta Informaticae 2001;41:187-228.

4. Jetzek F, Rahn CD, Dreschler-Fischer L. Ein geometrisches Modell für die Zellsegmentierung. Procs BVM 2006; 121-125.

5. Kass M, Witkin A, Terzopoulos D. Snakes: Active contour models. Int J Comp Vis 1988;1(4):321-331.

6. Wählby C, Lindblad J, Vondrus M, Bengtsson E, Björkesten L. Algorithms for cytoplasm segmentation of fluorescence labelled cells. Analytical Cellular Pathology $2002 ; 24(2-3): 101-111$. 